\title{
EXISTENCE AND UNIQUENESS RESULTS FOR DIRICHLET PROBLEM IN WEIGHTED SOBOLEV SPACES ON UNBOUNDED DOMAINS*
}

\author{
SERENA BOCCIA ${ }^{\dagger}$, MARIA SALVATO ${ }^{\dagger}$, AND MARIA TRANSIRICO ${ }^{\dagger}$
}

\begin{abstract}
In this paper we prove an existence and uniqueness theorem for the Dirichlet problem in $W_{s}^{2, p}$ for second order linear elliptic equations in unbounded domains. Here the leading coefficients are assumed to be locally $V M O$ and satisfy a suitable condition at infinity.
\end{abstract}

Key words. Elliptic equations, discontinuous coefficients, weighted spaces.

AMS subject classifications. 35J25, 35B65, 35R05.

1. Introduction. Let us consider the Dirichlet problem

$$
\left\{\begin{array}{l}
u \in W^{2, p}(\Omega) \cap \stackrel{\circ}{W}^{1, p}(\Omega) \\
L u=f, \quad f \in L^{p}(\Omega)
\end{array}\right.
$$

where $\Omega$ is a sufficiently regular open subset of $\left.\mathbb{R}^{n}(n \geq 3), p \in\right] 1,+\infty[, L$ is the uniformly elliptic second order linear differential operator defined by

$$
L=-\sum_{i, j=1}^{n} a_{i j} \frac{\partial^{2}}{\partial x_{i} \partial x_{j}}+\sum_{i=1}^{n} a_{i} \frac{\partial}{\partial x_{i}}+a
$$

with coefficients $a_{i j}=a_{j i} \in L^{\infty}(\Omega), i, j=1, \ldots, n$.

The problem (1.1) has been studied by several authors under various additional hypotheses on the $a_{i j}$ 's. In particular, a relevant existence and uniqueness theorem has been obtained in [6], [7], under the assumption that $\Omega$ is bounded, $a_{i j}$ 's are of class $V M O$ and $a_{i}=a=0$. This latter condition has been removed in [15], [16]. Recently, the above results have also been extended to the case of unbounded open sets (see [4], [5]).

More precisely, in [4], [5], assuming that $\Omega$ has the uniform $C^{1,1}$-regularity property, the leading coefficients satisfy similar restrictions to those in [6], [7] and the lower-order coefficients are in suitable spaces of Morrey type, the authors obtained certain a priori bounds for the solutions of (1.1). Using such estimates some existence and uniqueness results are established.

The aim of this paper is to study the problem (1.1) in certain weighted Sobolev spaces. Actually, we consider the following Dirichlet problem:

$$
\left\{\begin{array}{l}
u \in W_{s}^{2, p}(\Omega) \cap \stackrel{\circ}{W_{s}^{1, p}}(\Omega) \\
L u=f, f \in L_{s}^{p}(\Omega)
\end{array}\right.
$$

where $s \in \mathbb{R}, p \in] 1,+\infty\left[, W_{s}^{2, p}(\Omega), \stackrel{\circ}{W}_{s}^{1, p}(\Omega)\right.$ and $L_{s}^{p}(\Omega)$ are suitable weighted Sobolev spaces on an unbounded domain. Here, the hypotheses on the coefficients of the operator $L$ are similar to those required in [4], [5].

\footnotetext{
*Received November 26, 2009; accepted for publication May 12, 2011.

†Dipartimento di Matematica, Università di Salerno, via Ponte Don Melillo, I - 84084 Fisciano (SA), Italy (\{seboccia; msalvato; mtransirico\}@unisa.it).
} 
The class of weight functions we deal with is the set of all measurable functions $m: \Omega \rightarrow \mathbb{R}_{+}$such that

$$
\sup _{\substack{x, y \in \Omega \\|x-y|<d}} \frac{m(x)}{m(y)}<+\infty
$$

with $d \in \mathbb{R}_{+}$. Examples of functions verifying (1.4) are:

$$
m(x)=e^{t|x|}, \quad m(x)=\left(1+|x|^{2}\right)^{t}, \quad x \in \Omega, t \in \mathbb{R} .
$$

If $m$ satisfies the condition (1.4) and $k \in \mathbb{N}_{0}$, then $W_{s}^{k, p}(\Omega)$ denotes the space of distributions $u$ on $\Omega$ such that $m^{s} \partial^{\alpha} u \in L^{p}(\Omega)$ for $|\alpha| \leq k$, equipped with the norm

$$
\|u\|_{W_{s}^{k, p}(\Omega)}=\sum_{|\alpha| \leq k}\left\|m^{s} \partial^{\alpha} u\right\|_{L^{p}(\Omega)} .
$$

Moreover, $\stackrel{\circ}{W}_{s}^{k, p}(\Omega)$ denotes the closure of $C_{\circ}^{\infty}(\Omega)$ in $W_{s}^{k, p}(\Omega)$ and $L_{s}^{p}(\Omega)$ stands for $W_{s}^{0, p}(\Omega)$.

We note that the weight function $m^{s}$ has the role to fix the behaviour at infinity of the functions which lie in the weighted Sobolev space and of their derivatives.

We recall that in [3] it has been proved that there is a regularization function $\sigma$ verifying (1.4) too, which is equivalent to $m$ and such that:

$$
\sup _{x \in \Omega} \frac{\left|\partial^{\alpha} \sigma^{s}(x)\right|}{\sigma^{s}(x)}<+\infty \quad \forall \alpha \in \mathbb{N}_{0}^{n}, \quad \forall s \in \mathbb{R}
$$

Indeed, via the above condition (1.6), it has been proved that the map

$$
u \longrightarrow \sigma^{s} u
$$

defines a topological isomorphism from $W_{s}^{k, p}(\Omega)$ to $W^{k, p}(\Omega)$ and from $\stackrel{\circ}{W_{s}^{k, p}}(\Omega)$ to $\stackrel{\circ}{W}^{k, p}(\Omega)$. The last result allows to use, in [3], a priori no weighted estimates in [4], [5] to obtain a priori bounds for the solutions of the weighted problem (1.3). Since the lower terms are included in the operator $L$, in order to get such a priori bounds, it was necessary to study the multiplication operator

$$
u \in W_{s}^{k, p}(\Omega) \longrightarrow g u \in L_{s}^{p}(\Omega)
$$

and find conditions on the function $g$ which assure the boundedness or the compactness of (1.8).

In this paper, using an existence and uniqueness result for problem (1.1) (see [5]), the topological isomorphism (1.7) again, and an a priori estimate, obtained in [3], we are able to establish a uniqueness and existence theorem for problem (1.3).

A similar weighted problem was studied in [1], [2], with weight functions from a smaller class of that considered in this paper.

Acknowledgment. We wish to thank the referee for his useful comments. 
2. Notation and function spaces. Let $G$ be any Lebesgue measurable subset of $\mathbb{R}^{n}$ and $\Sigma(G)$ be the collection of all Lebesgue measurable subsets of $G$. For $F \in \Sigma(G)$, let $|F|$ denote the Lebesgue measure of $F$ and $\mathfrak{D}(F)$ the class of restrictions to $F$ of functions $\zeta \in C_{\circ}^{\infty}\left(\mathbb{R}^{n}\right)$ with $\bar{F} \cap \operatorname{supp} \zeta \subseteq F$. Moreover, if $X(F)$ is a space of functions defined on $F$, we denote by $X_{\text {loc }}(F)$ the class of all functions $g: F \rightarrow \mathbb{R}$ such that $\zeta g \in X(F)$ for any $\zeta \in \mathfrak{D}(F)$. Finally, for any $x \in \mathbb{R}^{n}$ and $r \in \mathbb{R}_{+}$, we put $B(x, r)=\left\{y \in \mathbb{R}^{n}:|y-x|<r\right\}, B_{r}=B(0, r)$ and $F(x, r)=F \cap B(x, r)$. Now let us recall the definitions of the function spaces in which the coefficients of the operator will be chosen. If $\Omega$ has the property

$$
\left.\left.|\Omega(x, r)| \geq A r^{n} \quad \forall x \in \Omega, \quad \forall r \in\right] 0,1\right]
$$

where $A$ is a positive constant independent of $x$ and $r$, then it is possible to consider the space $B M O(\Omega, \tau)\left(\tau \in \mathbb{R}_{+}\right)$of functions $g \in L_{\text {loc }}^{1}(\bar{\Omega})$ such that

$$
[g]_{B M O(\Omega, \tau)}=\sup _{\substack{x \in \Omega \\ r \in] 0, \tau]}} f_{\Omega(x, r)}\left|g-f_{\Omega(x, r)} g\right|<+\infty,
$$

where

$$
\int_{\Omega(x, r)} g=|\Omega(x, r)|^{-1} \int_{\Omega(x, r)} g .
$$

When $g \in B M O(\Omega)=B M O\left(\Omega, \tau_{A}\right)$, with

$$
\tau_{A}=\sup \left\{\tau \in \mathbb{R}_{+}: \sup _{\substack{x \in \Omega \\ r \in] 0, \tau]}} \frac{r^{n}}{|\Omega(x, r)|} \leq \frac{1}{A}\right\},
$$

we say that $g \in V M O(\Omega)$ if $[g]_{B M O(\Omega, \tau)} \rightarrow 0$ for $\tau \rightarrow 0^{+}$.

For $t \in\left[1,+\infty\left[\right.\right.$ and $\lambda \in\left[0, n\left[\right.\right.$, we denote by $M^{t, \lambda}(\Omega)$ the set of all functions $g$ in $L_{l o c}^{t}(\bar{\Omega})$ such that

$$
\|g\|_{M^{t, \lambda}(\Omega)}=\sup _{\substack{r \in j 0,1] \\ x \in \Omega}} r^{-\lambda / t}\|g\|_{L^{t}(\Omega(x, r))}<+\infty
$$

endowed with the norm defined by (2.2). Then we define $\tilde{M}^{t, \lambda}(\Omega)$ as the closure of $L^{\infty}(\Omega)$ in $M^{t, \lambda}(\Omega)$ and $M_{\circ}^{t, \lambda}(\Omega)$ as the closure of $C_{\circ}^{\infty}(\Omega)$ in $M^{t, \lambda}(\Omega)$. In particular, we put $M^{t}(\Omega)=M^{t, 0}(\Omega), \tilde{M}^{t}(\Omega)=\tilde{M}^{t, 0}(\Omega)$ and $M_{\circ}^{t}(\Omega)=M_{\circ}^{t, 0}(\Omega)$.

A more detailed account of properties of the above defined function spaces can be found in [11], [12] and [13].

3. Weight functions and weighted spaces. Let $\Omega$ be an open subset of $\mathbb{R}^{n}$ and let $d \in \mathbb{R}_{+}$. We are going to introduce a class of weight functions defined on $\Omega$. Indeed, denoted by $G_{d}(\Omega)$ the set of all measurable functions $m: \Omega \rightarrow \mathbb{R}_{+}$such that

$$
\sup _{\substack{x, y \in \Omega \\|x-y|<d}} \frac{m(x)}{m(y)}<+\infty
$$

then it is easy to verify that $m \in G_{d}(\Omega)$ if and only if there exists $\gamma \in \mathbb{R}_{+}$such that

$$
\gamma^{-1} m(y) \leq m(x) \leq \gamma m(y) \quad \forall y \in \Omega, \quad \forall x \in \Omega(y, d),
$$


where $\gamma \in \mathbb{R}_{+}$is independent of $x$ and $y$.

Observe that from (3.1) it follows

$$
m, m^{-1} \in L_{\mathrm{loc}}^{\infty}(\bar{\Omega}) .
$$

Now we define the class of weight functions in the following way:

$$
G(\Omega)=\bigcup_{d \in \mathbb{R}_{+}} G_{d}(\Omega)
$$

Examples of functions in $G(\Omega)$ are:

$$
m(x)=e^{t|x|}, \quad m(x)=\left(1+|x|^{2}\right)^{t}, \quad x \in \Omega, t \in \mathbb{R} .
$$

We can easily verify that if $m \in G(\Omega)$ then :

$$
m^{s} \in G(\Omega), \quad \forall s \in \mathbb{R} .
$$

Note that if $m \in G(\Omega)$ and $\Omega$ has the cone property, then it can be found a regularization function $\sigma \in G(\Omega) \cap C^{\infty}(\bar{\Omega})$ which is equivalent to $m$ and such that

$$
\left|\partial^{\alpha} \sigma(x)\right| \leq c_{\alpha} \sigma(x) \quad \forall x \in \Omega, \quad \forall \alpha \in \mathbb{N}_{0}^{n},
$$

where $c_{\alpha}$ is independent of $x$ (see Lemma 3.2 in [3]).

Some further interesting properties of the above defined weight functions can be found in [3].

Let $m$ be a function of class $G(\Omega)$. If $k \in \mathbb{N}_{0}, 1 \leq p<+\infty$ and $s \in \mathbb{R}$, consider the space $W_{s}^{k, p}(\Omega)$ of distributions $u$ on $\Omega$ such that $m^{s} \partial^{\alpha} u \in L^{p}(\Omega)$ for $|\alpha| \leq k$, equipped with the norm

$$
\|u\|_{W_{s}^{k, p}(\Omega)}=\sum_{|\alpha| \leq k}\left\|m^{s} \partial^{\alpha} u\right\|_{L^{p}(\Omega)} .
$$

Moreover, denote by $\stackrel{\circ}{W}_{s}^{k, p}(\Omega)$ the closure of $C_{\circ}^{\infty}(\Omega)$ in $W_{s}^{k, p}(\Omega)$ and put $W_{s}^{0, p}(\Omega)=L_{s}^{p}(\Omega)$. A more detailed account of properties of the above defined spaces can be found, for instance, in [14].

Now we consider the following condition:

$\left(h_{0}\right) \Omega$ has the cone property, $\left.p \in\right] 1,+\infty[, s \in \mathbb{R}, k, t$ are numbers such that:

$$
k \in \mathbb{N}, t \geq p, t \geq \frac{n}{k}, t>p \text { if } p=\frac{n}{k}, g \in M^{t}(\Omega) .
$$

From Theorem 3.1 of [9] we have the following.

THEOREM 3.1. If the assumption $\left(h_{0}\right)$ holds, then for any $u \in W_{s}^{k, p}(\Omega)$ we have $g u \in L_{s}^{p}(\Omega)$ and

$$
\|g u\|_{L_{s}^{p}(\Omega)} \leq c\|g\|_{M^{t}(\Omega)}\|u\|_{W_{s}^{k, p}(\Omega)}
$$


with $c$ depending only on $\Omega, n, k, p$ and $t$.

From now on, we will focus our attention on weight functions $m$ in $G(\Omega)$ such that:

$$
\begin{aligned}
& \lim _{|x| \rightarrow+\infty} m(x)=+\infty \\
\text { or } & \lim _{|x| \rightarrow+\infty} m(x)=0 .
\end{aligned}
$$

Without loss of generality, we can assume that only (3.6) holds. In fact, if the assumption (3.6) doesn't hold and then (3.7) holds we could give again the same proofs choosing like $\sigma$ the regularization function of the function $\frac{1}{m}$.

4. Tools. Let fix a cutoff function $f \in C_{\circ}^{\infty}\left(\overline{\mathbb{R}}_{+}\right)$such that

$$
0 \leq f \leq 1, \quad f(t)=1 \text { if } t \in[0,1], \quad f(t)=0 \text { if } t \in[2,+\infty[.
$$

Then we can define a sequence of functions $\left(\zeta_{k}\right)_{k \in \mathbb{N}}$ by

$$
\zeta_{k}: x \in \Omega \longrightarrow f\left(\frac{\sigma(x)}{k}\right) \quad \forall k \in \mathbb{N} .
$$

If $\Omega_{k}=\{x \in \Omega: \sigma(x)<k\}$, we easily have, for every $k \in \mathbb{N}$, that

$$
0 \leq \zeta_{k} \leq 1, \quad \zeta_{k}=1 \text { on } \bar{\Omega}_{k}, \quad \zeta_{k}=0 \text { on } \Omega \backslash \Omega_{2 k}, \quad \zeta_{k} \in C_{\circ}^{\infty}(\bar{\Omega}) .
$$

Now we can show that suitably combining the functions $\zeta_{k}$ and $\sigma$, we can determine a sequence of functions $\left(\eta_{k}\right)_{k \in \mathbb{N}}$, whose elements play a fundamental role in the sequel.

Let us define, for every $k \in \mathbb{N}$,

$$
\eta_{k}(x)=2 k \zeta_{k}(x)+\left(1-\zeta_{k}(x)\right) \sigma(x), \quad x \in \Omega .
$$

Simple calculations show that

$$
\begin{aligned}
\sigma(x) & \leq \eta_{k}(x), & & \text { if } x \in \bar{\Omega}_{2 k} \\
\eta_{k}(x) & \leq\left(1+c_{k}\right) \sigma(x), & & \text { if } x \in \bar{\Omega}_{2 k} \\
\sigma(x) & =\eta_{k}(x), & & \text { if } x \in \Omega \backslash \Omega_{2 k},
\end{aligned}
$$

where $c_{k} \in \mathbb{R}_{+}$depends only on $\mathrm{k}$.

So for any $k \in \mathbb{N}$, it holds that

$$
\sigma \sim \eta_{k}
$$

and

$$
\sigma^{s} \sim \eta_{k}^{s} \quad \forall s \in \mathbb{R}
$$


Moreover, for every $k \in \mathbb{N}$ the following estimates about derivatives hold

$$
\begin{array}{ll}
\left(\frac{\left(\eta_{k}\right)_{x}}{\eta_{k}}\right)(x)=\left(\frac{\left(\eta_{k}\right)_{x x}}{\eta_{k}}\right)(x)=0, & \text { if } x \in \Omega_{k} \\
\left(\frac{\left(\eta_{k}\right)_{x}}{\eta_{k}}\right)(x) \leq c_{1}\left(\frac{\sigma_{x}}{\sigma}\right)(x), & \text { if } x \in \Omega \backslash \Omega_{k} \\
\left(\frac{\left(\eta_{k}\right)_{x x}}{\eta_{k}}\right)(x) \leq c_{2}\left(\frac{\sigma_{x}^{2}+\sigma \sigma_{x x}}{\sigma^{2}}\right)(x), & \text { if } x \in \Omega \backslash \Omega_{k},
\end{array}
$$

and, more generally,

$$
\begin{array}{cc}
\left(\frac{\left(\eta_{k}\right)_{x}}{\eta_{k}}\right)(x) \leq c_{3} \sup _{x \in \Omega \backslash \Omega_{k}}\left(\frac{\sigma_{x}}{\sigma}\right)(x) & \forall x \in \Omega \\
\left(\frac{\left(\eta_{k}\right)_{x x}}{\eta_{k}}\right)(x) \leq c_{4} \sup _{x \in \Omega \backslash \Omega_{k}}\left(\frac{\sigma_{x}^{2}+\sigma \sigma_{x x}}{\sigma^{2}}\right)(x) & \forall x \in \Omega
\end{array}
$$

with $c_{1}, c_{2}, c_{3}$ and $c_{4}$ independent of $k$.

5. A uniqueness result. Assume that $\Omega$ is an unbounded open subset of $\mathbb{R}^{n}, n \geq 3$, with the uniform $C^{1,1}$-regularity property. Moreover, let $\left.p \in\right] 1,+\infty[$ and $s \in \mathbb{R}$. Consider in $\Omega$ the differential operator

$$
L=-\sum_{i, j=1}^{n} a_{i j} \frac{\partial^{2}}{\partial x_{i} \partial x_{j}}+\sum_{i=1}^{n} a_{i} \frac{\partial}{\partial x_{i}}+a
$$

with the following conditions on the coefficients:

$$
\left\{\begin{array}{l}
a_{i j}=a_{j i} \in L^{\infty}(\Omega) \cap V M O_{\mathrm{loc}}(\bar{\Omega}), i, j=1, \ldots, n, \\
\exists \nu>0: \sum_{i, j=1}^{n} a_{i j} \xi_{i} \xi_{j} \geq \nu|\xi|^{2} \quad \text { a.e. in } \Omega, \forall \xi \in \mathbb{R}^{n}
\end{array}\right.
$$

there exist functions $e_{i j}, i, j=1, \ldots, n, g$ and $\mu \in \mathbb{R}_{+}$such that

$\left(h_{2}\right) \quad\left\{\begin{array}{l}e_{i j}=e_{j i} \in L^{\infty}(\Omega), i, j=1, \ldots, n, \\ \left.\left.\left(e_{i j}\right)_{x_{h}} \in M_{\circ}^{t, n-t}(\Omega), \text { with } t \in\right] 2, n\right], \quad i, j, h=1, \ldots, n, \\ \sum_{i, j=1}^{n} e_{i j} \xi_{i} \xi_{j} \geq \mu|\xi|^{2} \text { a.e. in } \Omega, \quad \forall \xi \in \mathbb{R}^{n}, \\ g \in L^{\infty}(\Omega), \quad g_{0}=\underset{\Omega}{\operatorname{essinf} g>0, \quad g \in \operatorname{Lip}(\bar{\Omega}),} \\ \lim _{r \rightarrow+\infty} \sum_{i, j=1}^{n}\left\|e_{i j}-g a_{i j}\right\|_{L^{\infty}\left(\Omega \backslash B_{r}\right)}=0,\end{array}\right.$ 
$\left(h_{3}\right) \quad\left\{\begin{array}{l}a_{i} \in M_{\circ}^{t_{1}}(\Omega), i=1, \ldots, n, \\ a=a^{\prime}+b, a^{\prime} \in M_{\circ}^{t_{2}}(\Omega), b \in L^{\infty}(\Omega), b_{0}=\underset{\Omega}{\operatorname{ess} \inf b>0,} \\ a_{0}=\underset{\Omega}{\operatorname{ess} \inf } a>0,\end{array}\right.$

where

$$
\begin{aligned}
t_{1}>n & \text { if } p \leq n, \quad t_{1}=p \quad \text { if } p>n, \\
t_{2}>n / 2 \quad \text { if } p \leq n / 2, & t_{2}=p \quad \text { if } p>n / 2 .
\end{aligned}
$$

Observe that under assumptions $\left(h_{1}\right)-\left(h_{3}\right)$, the operator $L: W_{s}^{2, p}(\Omega) \rightarrow L_{s}^{p}(\Omega)$ is bounded from Theorem 3.1.

Adding the following assumption on the weight function

$$
\lim _{k \rightarrow+\infty} \sup _{\Omega \backslash \Omega_{k}} \frac{\sigma_{x}+\sigma_{x x}}{\sigma}=0,
$$

we can prove our uniqueness theorem.

Theorem 5.1. Assume $\left(h_{1}\right)-\left(h_{4}\right)$ true. Then the problem

$$
\left\{\begin{array}{l}
u \in W_{s}^{2, p}(\Omega) \cap \stackrel{\circ}{W}_{s}^{1, p}(\Omega) \\
L u=0
\end{array}\right.
$$

has only the zero solution.

Proof. From Theorem 4.3 of [5] and from the bounded inverse theorem (see Theorem 3.8 of [10]), there exists $c_{1} \in \mathbb{R}_{+}$such that

$$
\|u\|_{W^{2, p}(\Omega)} \leq c_{1}\|L u\|_{L^{p}(\Omega)} \quad \forall u \in W^{2, p}(\Omega) \cap \stackrel{\circ}{W^{1, p}}(\Omega) .
$$

Fix $u \in W_{s}^{2, p}(\Omega) \cap \stackrel{\circ}{W}_{s}^{1, p}(\Omega)$. Since $\eta_{k}^{s} u \in W^{2, p}(\Omega) \cap \stackrel{\circ}{W}^{1, p}(\Omega) \forall k \in \mathbb{N}$ (see Lemma 3.4 of [3]), from (5.3) then there exists $c_{2} \in \mathbb{R}_{+}$, independent of $u$ and $k$, such that

$$
\left\|\eta_{k}^{s} u\right\|_{W^{2, p}(\Omega)} \leq c_{2}\left\|L\left(\eta_{k}^{s} u\right)\right\|_{L^{p}(\Omega)} .
$$

For simplicity, in the sequel, we will write $\eta_{k}=\eta$. Since

$$
\begin{aligned}
L\left(\eta^{s} u\right) & =\eta^{s} L u-s \sum_{i, j=1}^{n} a_{i j}\left((s-1) \eta^{s-2} \eta_{x_{i}} \eta_{x_{j}} u+\eta^{s-1} \eta_{x_{i} x_{j}} u+\right. \\
& \left.+2 \eta^{s-1} \eta_{x_{i}} u_{x_{j}}\right)+s \sum_{i=1}^{n} a_{i} \eta^{s-1} \eta_{x_{i}} u
\end{aligned}
$$


from (5.4) and (5.5) we have:

$$
\begin{aligned}
\left\|\eta^{s} u\right\|_{W^{2, p}(\Omega)} & \leq c_{3}\left(\left\|\eta^{s} L u\right\|_{L^{p}(\Omega)}+\sum_{i, j=1}^{n}\left(\left\|\eta^{s-2} \eta_{x_{i}} \eta_{x_{j}} u\right\|_{L^{p}(\Omega)}+\right.\right. \\
& \left.+\left\|\eta^{s-1} \eta_{x_{i} x_{j}} u\right\|_{L^{p}(\Omega)}+\left\|\eta^{s-1} \eta_{x_{i}} u_{x_{j}}\right\|_{L^{p}(\Omega)}\right)+ \\
& \left.+\sum_{i=1}^{n}\left\|a_{i} \eta^{s-1} \eta_{x_{i}} u\right\|_{L^{p}(\Omega)}\right)
\end{aligned}
$$

where $c_{3} \in \mathbb{R}_{+}$is independent of $u$ and $k$. From Theorem 3.1 with $s=0$ and from (4.10) we get:

$$
\left\|a_{i} \eta^{s-1} \eta_{x_{i}} u\right\|_{L^{p}(\Omega)} \leq c_{4} \sup _{\Omega \backslash \Omega_{k}} \frac{\sigma_{x}}{\sigma}\left\|a_{i}\right\|_{M^{t_{1}}(\Omega)}\left\|\eta^{s} u\right\|_{W^{1, p}(\Omega)},
$$

where $c_{4}$ is independent of $u$ and $k$.

Thus, by (4.10), (4.11), (5.6) and (5.7), with easy computations, we obtain the bound:

$$
\begin{aligned}
\left\|\eta^{s} u\right\|_{W^{2, p}(\Omega)} & \leq c_{5}\left[\left\|\eta^{s} L u\right\|_{L^{p}(\Omega)}+\left(\sup _{\Omega \backslash \Omega_{k}} \frac{\sigma_{x}^{2}+\sigma \sigma_{x x}}{\sigma^{2}}+\right.\right. \\
& \left.\left.+\sup _{\Omega \backslash \Omega_{k}} \frac{\sigma_{x}}{\sigma}\right)\left\|\eta^{s} u\right\|_{W^{2, p}(\Omega)}\right]
\end{aligned}
$$

where $c_{5}$ is independent of $u$ and $k$.

By hypothesis $\left(h_{4}\right)$, there exists $k_{0} \in \mathbb{N}$ such that:

$$
\left(\sup _{\Omega \backslash \Omega_{k_{0}}} \frac{\sigma_{x}^{2}+\sigma \sigma_{x x}}{\sigma^{2}}+\sup _{\Omega \backslash \Omega_{k_{0}}} \frac{\sigma_{x}}{\sigma}\right) \leq \frac{1}{2 c_{5}} .
$$

Now, if we denote with $\eta$ the function $\eta_{k_{0}}$, from (5.8) and (5.9) we can deduce that:

$$
\left\|\eta^{s} u\right\|_{W^{2, p}(\Omega)} \leq c_{6}\left\|\eta^{s} L u\right\|_{L^{p}(\Omega)},
$$

and then, using (4.9), from (5.10) we obtain that:

$$
\|u\|_{W_{s}^{2, p}(\Omega)} \leq c_{7}\|L u\|_{L_{s}^{p}(\Omega)},
$$

with $c_{6}, c_{7}$ independent of $u$, and then the claimed result.

6. Existence results. The aim of this section is to establish some existence results concerning the problem (1.3). We start with a lemma which we will need in the proof of our main existence result.

LEMMA 6.1. Let

$$
L_{0}=-\sum_{i, j=1}^{n} a_{i j} \frac{\partial^{2}}{\partial x_{i} \partial x_{j}}
$$


and assume that $\left(h_{1}\right),\left(h_{2}\right),\left(h_{4}\right)$ hold. Then the Dirichlet problem

$$
\left\{\begin{array}{l}
u \in W_{s}^{2, p}(\Omega) \cap \stackrel{\circ}{W}_{s}^{1, p}(\Omega) \\
L_{0} u+c u=f, \quad f \in L_{s}^{p}(\Omega)
\end{array}\right.
$$

where

$$
c=1+\left|-s(s+1) \sum_{i, j=1}^{n} a_{i j} \frac{\sigma_{x_{i}}}{\sigma} \frac{\sigma_{x_{j}}}{\sigma}+s \sum_{i, j=1}^{n} a_{i j} \frac{\sigma_{x_{i} x_{j}}}{\sigma}\right|,
$$

is uniquely solvable.

Proof. Note that $u$ is a solution of the problem (6.1) if and only if $w=\sigma^{s} u$ is a solution of the problem

$$
\left\{\begin{array}{l}
w \in W^{2, p}(\Omega) \cap \stackrel{\circ}{W^{1, p}}(\Omega) \\
-\sum_{i, j=1}^{n} a_{i j}\left(\sigma^{-s} w\right)_{x_{i} x_{j}}+c \sigma^{-s} w=f, \quad f \in L_{s}^{p}(\Omega) .
\end{array}\right.
$$

Since, for any $i, j \in\{1, \ldots, n\}$

$$
\begin{aligned}
\frac{\partial^{2}}{\partial x_{i} \partial x_{j}}\left(\sigma^{-s} w\right) & =\sigma^{-s} w_{x_{i} x_{j}}-2 s \sigma^{-s-1} \sigma_{x_{i}} w_{x_{j}}+s(s+1) \sigma^{-s-2} \sigma_{x_{i}} \sigma_{x_{j}} w+ \\
& -s \sigma^{-s-1} \sigma_{x_{i} x_{j}} w
\end{aligned}
$$

then (6.3) is equivalent to the problem

$$
\left\{\begin{array}{l}
w \in W^{2, p}(\Omega) \cap \stackrel{\circ}{W^{1, p}}(\Omega) \\
L_{0} w+\sum_{i=1}^{n} \alpha_{i} w_{x_{i}}+\alpha w=\sigma^{s} f
\end{array}\right.
$$

where:

$$
\begin{gathered}
\alpha_{i}=2 s \sum_{j=1}^{n} a_{i j} \frac{\sigma_{x_{j}}}{\sigma}, \quad i=1, \ldots, n, \\
\alpha=c-s(s+1) \sum_{i, j=1}^{n} a_{i j} \frac{\sigma_{x_{i}}}{\sigma} \frac{\sigma_{x_{j}}}{\sigma}+s \sum_{i, j=1}^{n} a_{i j} \frac{\sigma_{x_{i} x_{j}}}{\sigma} .
\end{gathered}
$$

By Theorem 4.3 of [5], (1.6) of [11] and (3.3), we obtain that (6.4) is uniquely solvable and then the problem (6.1) is uniquely solvable too. $\square$

TheOREM 6.2. Suppose that conditions $\left(h_{1}\right)-\left(h_{4}\right)$ hold. Then the problem

$$
\left\{\begin{array}{l}
u \in W_{s}^{2, p}(\Omega) \cap \stackrel{\circ}{W}_{s}^{1, p}(\Omega) \\
L u=f, \quad f \in L_{s}^{p}(\Omega)
\end{array}\right.
$$


is uniquely solvable.

Proof. For each $\tau \in[0,1]$ put

$$
L_{\tau}=\tau L+(1-\tau)\left(L_{0}+c\right),
$$

where $c$ is the function defined by (6.2). The operator

$$
\tau \in[0,1] \longmapsto L_{\tau} \in B\left(W_{s}^{2, p}(\Omega) \cap \stackrel{\circ}{W_{s}^{1, p}}(\Omega), L_{s}^{p}(\Omega)\right)
$$

is clearly continuous. By Theorem 5.2 of [3] and Theorem 5.1 we can say that the operator $L_{\tau}$ has closed range and null kernel. Now, by Lemma 4.1 of [5], there exists a positive real number $c_{0}$ such that

$$
\begin{aligned}
& \|u\|_{W_{s}^{2, p}(\Omega)} \leq c_{0}\left\|L_{\tau} u\right\|_{L_{s}^{p}(\Omega)}, \\
& \forall u \in W_{s}^{2, p}(\Omega) \cap \stackrel{\circ}{W}_{s}^{1, p}(\Omega), \quad \forall \tau \in[0,1] .
\end{aligned}
$$

Using Lemma 6.1, the problem

$$
\left\{\begin{array}{l}
u \in W_{s}^{2, p}(\Omega) \cap \stackrel{\circ}{W}_{s}^{1, p}(\Omega) \\
L_{0} u+c u=f, \quad f \in L_{s}^{p}(\Omega)
\end{array}\right.
$$

is uniquely solvable.

Therefore, this latter result and the estimate (6.6) allow to use the method of continuity along a parameter (see, e.g., Theorem 5.2 of [8]) in order to prove that the problem

$$
\left\{\begin{array}{l}
u \in W_{s}^{2, p}(\Omega) \cap \stackrel{\circ}{W}_{s}^{1, p}(\Omega) \\
L u=f, \quad f \in L_{s}^{p}(\Omega)
\end{array}\right.
$$

is likewise uniquely solvable. The proof is now complete.

\section{REFERENCES}

[1] S. Boccia, S. Monsurrò, and M. Transirico, Elliptic equations in weighted Sobolev spaces on unbounded domains, Int. J. Math. Math. Sci. (2008), ID 582435, 12 pages.

[2] S. Boccia, S. Monsurrò, And M. Transirico, Solvability of the Dirichlet problem for elliptic equations in weighted Sobolev spaces on unbounded domains, Bound. Value Probl. (2008), ID 901503, 13 pages.

[3] S. Boccia, M. Salvato, and M. Transirico, A Priori Bounds for Elliptic Operators in Weighted Sobolev Spaces, J. Math. Inequal., to appear.

[4] L. Caso, P. Cavaliere, and M. Transirico, Uniqueness results for elliptic equations with VMO - coefficients, Int. J. Pure Appl. Math., 13 (2004), pp. 499-512.

[5] L. Caso, P. Cavaliere, and M. Transirico, An existence result for elliptic equations with VMO - coefficients, J. Math. Anal. Appl., 325 (2007), pp. 1095-1102.

[6] F. Chiarenza, M. Frasca, And P. Longo, Interior $W^{2, p}$ estimates for non divergence elliptic equations with discontinuous coefficients, Ricerche Mat., 40 (1991), pp. 149-168.

[7] F. Chiarenza, M. Frasca, And P. Longo, $W^{2, p}$-solvability of the Dirichlet problem for nondivergence elliptic equations with VMO coefficients, Trans. Amer. Math. Soc., 336 (1993), pp. 841-853. 
[8] D. Gilbarg and N. S. Trudinger, Elliptic Partial Differential Equations of Second Order, Reprint of the 1998 ed., Springer, Berlin, 2001.

[9] A. V. Glushak, M. Transirico, and M. Troisi, Teoremi di immersione ed equazioni ellittiche in aperti non limitati, Rend. Mat. (7), 9 (1989), pp. 113-130.

[10] M. Schechter, Principles of Functional Analysis, American Mathematical Society, Providence, RI, (2002).

[11] M. Transirico And M. Troisi, Equazioni ellittiche del secondo ordine di tipo non variazionale in aperti non limitati, Ann. Mat. Pura Appl. (4), 152 (1988), pp. 209-226.

[12] M. Transirico, M. Troisi, and A. Vitolo, Spaces of Morrey type and elliptic equations in divergence form on unbounded domains, Boll. Un. Mat. Ital. (7), 9-B (1995), pp. 153-174.

[13] M. Transirico, M. Troisi, and A. Vitolo, BMO spaces on domains of $\mathbb{R}^{n}$, Ricerche Mat., 45 (1996), pp. 355-378.

[14] M. Troisi, Su una classe di spazi di Sobolev con peso, Rend. Accad. Naz. Sci. XL Mem. Mat., 10 (1986), pp. 177-189.

[15] C. Vitanza, $W^{2, p}$-regularity for a class of elliptic second order equations with discontinuous coefficients, Matematiche (Catania), 47 (1992), pp. 177-186.

[16] C. Vitanza, A new contribution to the $W^{2, p}$-regularity for a class of elliptic second order equations with discontinuous coefficients, Matematiche (Catania) 48 (1993), pp. 287-296. 
